\title{
QGP thermodynamics and Meson spectroscopy with AdS/CFT
}

\author{
Johanna Erdmenger, Felix Rust* \\ Max-Planck-Institut für Physik (Werner-Heisenberg-Institut), Munich, Germany \\ E-mail: jke@mppmu.mpg.de, rust @mppmu.mpg.de
}

\section{Matthias Kaminski}

Instituto de Fisica Teórica, UAM/CSIC, Madrid, Spain

E-mail: matthias.kaminskiduam.es

In this talk we present applications of the AdS/CFT correspondence to strongly coupled systems at finite temperature and particle density. The model we investigate contains adjoint matter described by the gauge multiplet of $\mathscr{N}=4$, as well as fundamental matter given by the hypermultiplet of $\mathscr{N}=2$ Super Yang-Mills theory. In some aspects these systems can be thought of as models for the quark-gluon plasma.

In the first part we review some properties of meson spectra obtained from these holographic models. We discuss the implications of finite temperature and particle density in these stringtheory motivated setups. In particular, we find a broadening of the vector meson peaks in the relevant spectral function at finite density. However, we do not observe a movement of the resonances to lower frequencies.

Moreover, we analyze the effects of strong coupling on heavy meson diffusion in medium. To do so we make use of an effective model with dipole interaction, which is valid for heavy quarks at arbitrary coupling strength. We calculate the momentum broadening - normalized to the in-medium mass shift — and compare the large 't Hooft coupling AdS/CFT result with a perturbative result for weak coupling. We find that the momentum broadening is reduced at large 't Hooft coupling, leading to increased relaxation time.

8th Conference Quark Confinement and the Hadron Spectrum

September 1-6, 2008

Mainz. Germany

\footnotetext{
* Speaker.
} 


\section{Introduction}

The AdS/CFT correspondence maps string theory, or certain limits thereof, to quantum field theory. A well-established explicit formulation maps classical supergravity to large $N$ gauge theory. Application of the AdS/CFT correspondence to strongly coupled systems at finite temperature and particle density is interesting from two points of view. First, holographic models allow for qualitative comparison of string-theory motivated calculations with phenomenology. Second, the AdS/CFT correspondence provides a very useful approach to dynamical processes in strongly coupled field theories. Holographic models therefore may prove to be valuable tools to gain insight into the nature of matter under extreme conditions, as it existed shortly after the big bang or in heavy ion collisions.

We pursue the strategy of the top-down approach. This means we use solutions of tendimensional supergravity to investigate the properties of the dual field theories. In this way we restrict our attention to the best accessible field theory limits, i. e. asymptotic low energy, large number $N$ of color degrees of freedom and strong coupling.

In particular we are interested in bound states of fundamental and anti-fundamental matter described by the hypermultiplet of $\mathscr{N}=2$ Super Yang-Mills (SYM) theory, accompanied by gauge fields in the adjoint gauge multiplet of $\mathscr{N}=4$ SYM theory. We interpret these bound states as quark-antiquark mesons in a quark-gluon plasma. In the quantum field theory, the vector valued bound states of quarks can be described by the retarded two-point correlation function $G^{R}$ of flavor currents $J$,

$$
G^{R}(\omega)=-i \int \mathrm{d}^{4} x e^{i \vec{k} \vec{x}} \theta\left(x^{0}\right)\langle[J(\overrightarrow{0}), J(\vec{x})]\rangle .
$$

Generalizations of the AdS/CFT correspondence allow to compute these correlators at strong coupling, and to derive meson mass spectra from them.

The same methods can be used to estimate the effect of strong coupling on meson diffusion. The coupling constant in our effective model is determined by the quotient $\lambda / m_{q}$ of 't Hooft coupling and quark mass. We compare momentum broadening at strong coupling, obtained from AdS/CFT calculations, to perturbative results at weak coupling. These efforts are motivated by the search for a mechanism of the suppression of heavy quarks observed at RHIC, and energy loss of mesons in the strongly coupled medium.

\section{Holographic meson spectra at finite temperature and particle density}

We work in the D3/D7 setup, where a finite small number of $N_{f}$ D7-branes is embedded into the $\mathrm{AdS}_{5} \times \mathrm{S}^{5}$ black hole background, which is generated by a stack of $N \gg N_{f}$ D3-branes. We restrict to the low energy limit, where the resulting degrees of freedom of string excitations are dual to the $\mathscr{N}=4$ gauge multiplet plus the $\mathscr{N}=2$ hypermultiplet of SYM theory. We mainly focus on the latter degrees of freedom which transform in the fundamental representation of $\mathrm{SU}(N)$ and $\mathrm{SU}\left(N_{f}\right)$. We may therefore interpret $N$ as the number of colors, $N_{f}$ as the number of flavors, and the hypermultiplet as the supersymmetric realization of quark fields.

According to the AdS/CFT dictionary, the parameters and fields of the gravity setup translate into field theory equivalents as follows. The AdS black hole radius is proportional to the field 
theory temperature $T$, which breaks supersymmetry. The separation of the D3- and D7-branes is proportional to the quark mass $m_{q}$, which breaks conformal symmetry. We normalize dimensionful quantities to the temperature and use $m \propto m_{q} / T$ as a free parameter. The second free parameter is the particle density $\tilde{d}$, which is proportional to the value of the time component of the $\operatorname{SU}\left(N_{f}\right)$ valued gauge field $A$ in the supergravity theory, evaluated on the boundary of $\operatorname{AdS}_{5}$ space. Additional to the flavor symmetry group $\mathrm{SU}\left(N_{f}\right)$ the full symmetry group $\mathrm{U}\left(N_{f}\right) \cong \mathrm{U}(1) \times \mathrm{SU}(N)$ of the brane configuration also exhibits a $U(1)$ factor. We work in $N_{f}=2$ and interpret the U(1) charge as baryon number and the $\mathrm{SU}(2)$ charge as isospin number. In this way we can tune the baryon and isospin particle density $\tilde{d}$ by specifying the boundary value of $A$.

To derive the spectra of bound states of quark-antiquark pairs as above, we need to determine the embedding of the D7-branes as well as the gauge field configuration on the brane. They are determined by extremizing the Dirac-Born-Infeld action, which is given by

$$
S_{\mathrm{DBI}}=-T_{7} \int \mathrm{d}^{8} \xi \sqrt{G+2 \pi \alpha^{\prime} F} .
$$

Here the D7-brane embeddings determine the induced metric $G$ of the D7-brane, and $F=\mathrm{d} A$ is the field strength tensor of the gauge field on the brane, and $T_{7}$ is the brane tension.

We describe the field theory meson spectrum in terms of the spectral function $\Re$, defined as

$$
\mathfrak{R}(\omega)=-2 \operatorname{Im} G^{R}(\omega) .
$$

To obtain $G^{R}$, as defined in (1.1), we make use of the AdS/CFT correspondence which allows us to identify the partition sum of the D3/D7 setup with the generating functional of the dual field theory. The partition function is given by $Z=\exp S_{\mathrm{DBI}}$. As the boundary value of the gauge field $A$ is the source of the operator $J$ in (1.1), we can obtain correlation functions by evaluating functional derivatives of the above partition function [1]. Explicit calculation yields

$$
G^{R}=\frac{N_{f} N T^{2}}{8} \lim _{\rho \rightarrow \infty}\left(\rho^{3} \frac{\partial_{\rho} A}{A}\right)
$$

where we have to insert $A$, which we obtain as a numerical solution to the equations of motion derived from the DBI action (2.1) [2].

The resulting vector meson spectra depending on the normalized frequency $\mathfrak{w}=\omega /(2 \pi T)$ for $N_{f}=2$ are shown in figures 1,2 and 3. In figure 1 we compare the obtained spectra for a certain choice of quark mass/temperature ratio and particle density to the analytically known line spectrum for the case of zero temperature and vanishing particle density, where supersymmetry is restored. At low temperatures we observe resonance peaks in the derived spectra in accordance with the expectations from supersymmetric setups. Moreover we also see the phenomenologically expected behavior of finite resonance peak widths, indicating finite lifetimes of the bound states at finite temperature, referred to as meson melting. Figure 2 shows the influence of variations in the particle density. Increasing the density destabilizes the bound states and slightly shifts the positions of the resonance peaks to higher energies. Such in-medium effects have also been observed in effective field theory models [3]. We do not observe Brown-Rho scaling, which predicts decreasing meson masses [4]. Finally, figure 3 shows the splitting of the vector meson spectrum at finite isospin density. The isospin $1 / 2$ quarks combine to the different possible combinations constituting an isospin 1 meson triplet with $z$ components $-1,0$ and +1 , respectively. 


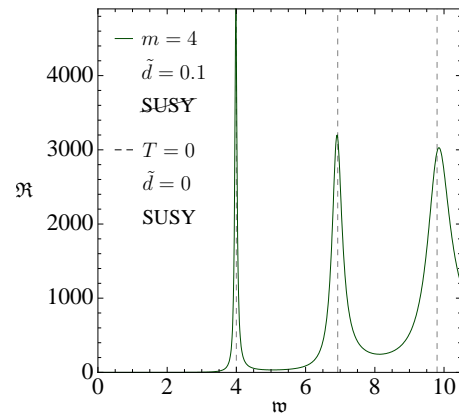

Figure 1: At low temperatures the spectrum coincides with the exact supersymmetric result.

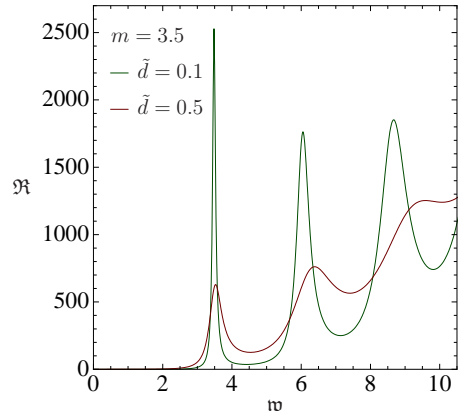

Figure 2: At increasing baryon density the resonances are broadened and slightly shifted.

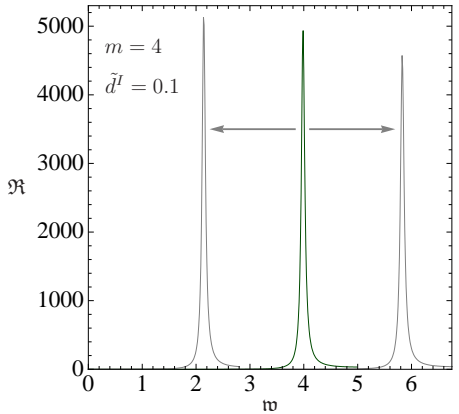

Figure 3: At finite isospin density the peaks split up to form an isospin triplet.

\section{Quarkonium diffusion}

We make use of the effective Lagrangian from [5] for the heavy meson field $\phi$ with mass $M$. Transcription to $\mathscr{N}=4 \mathrm{SYM}$ theory in lowest order in $T / M$ yields

$$
\mathscr{L}=-i \phi^{\dagger} \partial \phi+\frac{c_{T}}{N^{2}} \phi^{\dagger} \mathscr{T}^{00} \phi+\frac{c_{F}}{N^{2}} \phi^{\dagger} \mathscr{O}_{F^{2}} \phi .
$$

We refer to the last two terms as the interaction Lagrangian $\mathscr{L}_{\text {int }}$, where $\mathscr{T}^{00}=\frac{3}{8} \pi^{2} N^{2} T^{4}$ is the 00-component of the energy momentum tensor and $\mathscr{O}_{F^{2}}=\operatorname{tr} F^{2}$ is the operator which mediates the coupling of the mesons to the gauge bosons. The coefficients $c_{T}$ and $c_{F}$ determine the mass shift of the mesons in medium by $\delta M=-\left\langle\mathscr{L}_{\text {int }}\right\rangle=-\left(c_{E}\left\langle\mathscr{T}^{00}\right\rangle+c_{F}\left\langle\mathscr{O}_{F^{2}}\right\rangle\right) / N^{2}$.

In a Langevin process the diffusion of the mesons in the medium can be described by the momentum broadening $\kappa=2 \pi M T \eta$, which determines the change of momentum $\vec{p}$ by damping and a random force $\vec{\xi}$ (random kicks) by

$$
\frac{\mathrm{d} p_{i}}{\mathrm{~d} t}=\xi_{i}(t)-\eta p_{i}, \quad \text { with } \quad\left\langle\xi_{i}(t) \xi_{j}\left(t^{\prime}\right)\right\rangle=\kappa \delta_{i j} \delta\left(t-t^{\prime}\right) .
$$

On time scales short compared to equilibration times but long compared to medium correlations we have

$$
\kappa \propto \int \mathrm{d} t \int \mathrm{d} t^{\prime}\left\langle\xi_{i}(t) \xi_{j}\left(t^{\prime}\right)\right\rangle=\int \mathrm{d} t\left\langle\frac{\mathrm{d} p_{i}}{\mathrm{~d} t} \frac{\mathrm{d} p_{j}}{\mathrm{~d} t}\right\rangle=\int \mathrm{d} t\left\langle\mathscr{F}_{i} \mathscr{F}_{j}\right\rangle .
$$

We derive the force $\mathscr{F}$ from the interaction Lagrangian $\mathscr{L}_{\text {int }}$, given by the last two terms in (3.1), $\mathscr{F}=-\int \mathrm{d}^{3} x \phi^{\dagger} \nabla \mathscr{L}_{\text {int }} \phi$. Plugging this into (3.3) leads to

$$
\kappa=-\lim _{\omega \rightarrow \infty} \int \frac{\mathrm{d}^{3} q}{(2 \pi)^{3}} \frac{2 T q^{2}}{3 \omega N^{4}}\left(c_{T}^{2} \operatorname{Im} G_{\mathscr{T}}^{R}+c_{F}^{2} \operatorname{Im} G_{F^{2}}^{R}\right) .
$$

The two-point correlation functions $G^{R}$ of the energy momentum tensor and the field strength tensor can be obtained perturbatively at weak coupling, and via AdS/CFT at strong coupling in a similar way as above. The same applies for the mass shift $\delta M$. The quotient $\kappa /(\delta M)^{2}$ does not depend on the coefficients $c_{E}$ and $c_{F}$. We can compare this quantity in the limit of weak coupling with the result at asymptotically strong coupling [6],

$$
\left[\frac{\kappa}{(\delta M)^{2}}\right]_{\lambda \rightarrow 0}=\frac{\pi T}{N^{2}} 37.0, \quad\left[\frac{\kappa}{(\delta M)^{2}}\right]_{\lambda \rightarrow \infty}=\frac{\pi T}{N^{2}} 8.3 .
$$


We thus see that the momentum broadening is reduced in the limit of strong coupling, leading to increased diffusion and relaxation time.

\section{Conclusion}

Using the example of the D3/D7 system, we showed that holographic models are capable of describing spectra of meson-like bound states of supersymmetric fundamental degrees of freedom. These spectra exhibit many qualitative features expected in finite temperature, finite density strongly coupled QCD. Among these are increased decay rates at high temperature, in-medium effects of increased decay rates and mass shifts at finite particle density, as well as the triplet splitting of vector meson spectra at finite isospin density.

In an effective Lagrangian model we used holographic techniques to estimate strong coupling effects on meson diffusion in a model of the strongly coupled quark-gluon plasma. The results suggest that the drag forces on mesons in the medium are reduced at strong coupling, such that momentum broadening in the medium is reduced and relaxation times increase. In this sense the observed suppression of the quarkonium drag coefficient is analog to the suppression of the shear viscosity coefficient at strong coupling.

\section{Acknowledgments}

We are grateful to D. Teaney, K. Dusling and C. Young for fruitful collaboration. We thank the organizers of the 8th Conference on Quark Confinement and the Hadron Spectrum for an enriching congress. Part of this work was funded by the Cluster of Excellence for Fundamental PhysicsOrigin and Structure of the Universe.

\section{References}

[1] D. T. Son and A. O. Starinets, Minkowski-space correlators in AdS/CFT correspondence: Recipe and applications, JHEP 09 (2002) 042, [arXiv:hep-th/0205051].

[2] J. Erdmenger, M. Kaminski, and F. Rust, Holographic vector mesons from spectral functions at finite baryon or isospin density, Phys. Rev. D77 (2008) 046005, [arXiv:0710.0334].

[3] R. Rapp and J. Wambach, Low mass dileptons at the CERN-SPS: Evidence for chiral restoration?, Eur. Phys. J. A6 (1999) 415-420, [arXiv:hep-ph/9907502].

[4] G. E. Brown and M. Rho, Scaling effective Lagrangians in a dense medium, Phys. Rev. Lett. 66 (1991) $2720-2723$.

[5] M. E. Luke, A. V. Manohar, and M. J. Savage, A QCD Calculation of the interaction of quarkonium with nuclei, Phys. Lett. B288 (1992) 355-359, [arXiv:hep-ph/9204219].

[6] K. Dusling, J. Erdmenger, M. Kaminski, F. Rust, D. Teaney, and C. Young, Quarkonium transport in thermal AdS/CFT, JHEP 10 (2008) 098, [arXiv:0808.0957]. 